\title{
Advanced Unsupervised Classification Methods to Detect Anomalies on Earthen Levees Using Polarimetric SAR Imagery
}

\author{
Ramakalavathi Marapareddy ${ }^{1, *}$, James V. Aanstoos ${ }^{2}$ and Nicolas H. Younan ${ }^{3}$ \\ 1 Center for Advanced Vehicular Systems, Mississippi State University, Mississippi State, MS 39759, USA \\ 2 Geosystems Research Institute, Mississippi State University, Mississippi State, MS 39759, USA; \\ aanstoos@gri.msstate.edu \\ 3 Department of Electrical and Computer Engineering, Mississippi State University, Mississippi State, \\ MS 39762, USA; younan@ece.msstate.edu \\ * Correspondence: kala@cavs.msstate.edu; Tel.: +1-662-325-2057
}

Academic Editor: Assefa M. Melesse

Received: 3 April 2016; Accepted: 6 June 2016; Published: 16 June 2016

\begin{abstract}
Fully polarimetric Synthetic Aperture Radar (polSAR) data analysis has wide applications for terrain and ground cover classification. The dynamics of surface and subsurface water events can lead to slope instability resulting in slough slides on earthen levees. Early detection of these anomalies by a remote sensing approach could save time versus direct assessment. We used L-band Synthetic Aperture Radar (SAR) to screen levees for anomalies. SAR technology, due to its high spatial resolution and soil penetration capability, is a good choice for identifying problematic areas on earthen levees. Using the parameters entropy $(\mathrm{H})$, anisotropy $(\mathrm{A})$, alpha $(\alpha)$, and eigenvalues $\left(\lambda, \lambda_{1}, \lambda_{2}\right.$, and $\lambda_{3}$ ), we implemented several unsupervised classification algorithms for the identification of anomalies on the levee. The classification techniques applied are $\mathrm{H} / \alpha, \mathrm{H} / \mathrm{A}, \mathrm{A} / \alpha$, Wishart $\mathrm{H} / \alpha$, Wishart $\mathrm{H} / \mathrm{A} / \alpha$, and $\mathrm{H} / \alpha / \lambda$ classification algorithms. In this work, the effectiveness of the algorithms was demonstrated using quad-polarimetric L-band SAR imagery from the NASA Jet Propulsion Laboratory's (JPL's) Uninhabited Aerial Vehicle Synthetic Aperture Radar (UAVSAR). The study area is a section of the lower Mississippi River valley in the Southern USA, where earthen flood control levees are maintained by the US Army Corps of Engineers.
\end{abstract}

Keywords: classification; earthen levees; radar polarimetry; Synthetic Aperture Radar; UAVSAR

\section{Introduction}

Earthen levees protect large areas of populated and cultivated land in the United States from flooding. The potential loss of life and property associated with the catastrophic failure of levees can be extremely large. Over the entire US, there are more than $150,000 \mathrm{~km}$ of levee structures of varying designs and conditions. One type of problem along these levees, which can contribute to failure during a high water event, is the occurrence of slough slides [1]. Slough (or slump) slides are slope failures along a levee, which leave areas of the levee vulnerable to seepage and failure during high water events [2]. The roughness and related textural characteristics of the soil in a slide area affect the amount and pattern of radar backscatter. The type of vegetation that grows in a slide area differs from the surrounding levee vegetation, which can also be used in detecting slides [3].

Polarimetric Synthetic Aperture Radar (PolSAR) data encompass information on scattering mechanisms by diverse target structures and materials. We used multi-polarized L-band Synthetic Aperture Radar (SAR) to screen earthen levees for anomalies. The dynamics of surface and subsurface water events can lead to slope instability resulting in slough slides [4]. If these levees are not healthy, 
they may not be able to withstand flood conditions which could lead to catastrophic failures. Improved knowledge of the condition of these levees would significantly improve the allocation of precious resources to inspect, test, and repair the ones most in need [5]. Early detection of these anomalies by a remote sensing approach could save time versus direct assessment. SAR technology, due to its high spatial resolution and soil penetration capability, is a good choice for identifying problematic areas on levees for this purpose [5]. SAR polarimetry using quad-polarization data is the HV-polarization base in which an antenna transmits and receives horizontally and vertically polarized signals [6].

$\mathrm{H} / \mathrm{A} / \alpha$ decomposition is an eigenvalue-based decomposition of the coherency matrix $\left\langle\left[T_{3}\right]\right\rangle[7,8]$. Three features are defined as a function of the eigenvalues and the eigenvectors of $\left\langle\left[T_{3}\right]\right\rangle:$ (1) entropy $(\mathrm{H})$, which determines the randomness of scattering or degree of statistical disorder of target; (2) anisotropy (A), which is a unique function of eigenvalue ratios; (3) mean alpha angle $(\alpha)$ for different scattering processes and identifying the dominant scattering mechanism [9,10]; and lambda $(\lambda)$ defined as nonnegative real eigenvalues of the diagonal matrix $\left[\Sigma_{3}\right][7,8]$. Cloude and Pottier [9] demonstrated an unsupervised classification based on the $\mathrm{H} / \alpha$ parameters. These parameters alone were not sufficient for good interclass resolution, indicating that additional information is needed. Hellmann et al. [11] tested an unsupervised classification based on the $H / \alpha / \lambda_{1}$ parameters. However, $\lambda_{1}$ alone was not able to represent the complete scattering mechanism about the target. Lee $e t$ al. [12] proposed an unsupervised approach using $\mathrm{H}$ and $\alpha$ to initially classify a SAR image and use this classification as training data for a final supervised classification using the maximum likelihood algorithm base on a Wishart distribution. Further improvements can also be made by using the anisotropy parameter. The value of the anisotropy gives the relative significance of secondary scattering mechanisms.

\section{Method}

The overall method consists of creating an image subset of the test area, testing our candidate classifiers on the area of interest, and comparing the results to ground truth data. In this paper, we implemented several unsupervised classification algorithms for the identification of anomalies such as slough slides on the levee, an example of which is shown in Figure 1. The classification techniques applied, using $\mathrm{H}, \mathrm{A}, \alpha$, and $\lambda$ parameters are $\mathrm{H} / \alpha, \mathrm{H} / \mathrm{A}$, and $\mathrm{A} / \alpha$ classification $[9,10]$, Wishart $\mathrm{H} / \alpha$ classification [13], Wishart $\mathrm{H} / \mathrm{A} / \alpha$ classification [14], and extended $\mathrm{H} / \alpha$ (i.e., $\mathrm{H} / \alpha / \lambda$ ) classification [11,14,15], including classification for individual $\lambda$ values as $H / \alpha / \lambda_{1}, H / \alpha / \lambda_{2}$, and $\mathrm{H} / \alpha / \lambda_{3}$. The $\mathrm{H} / \alpha$ two dimensional classification employs a three-level Bernoulli statistical model to generate estimates of the average target scattering matrix parameters from the data [9]. The work outlined here is also focused on using $\lambda_{1}, \lambda_{2}$, and $\lambda_{3}$, which takes advantage of individual classification using $\lambda_{1}, \lambda_{2}$, and $\lambda_{3}$ for a good interclass resolution. In the $H / \alpha / \lambda$ approach, the backscatter intensity information contained in the eigenvalues $\lambda_{1}, \lambda_{2}$, and $\lambda_{3}$ is used to improve the interclass resolution due to the different reflectivities of different scatterers. The classification is performed using the complex data of the Multi-Look Cross products (MLC) acquired by UAVSAR. The MLC data is derived from an average of 3 pixels in range and 12 pixels in azimuth of the single-look complex data (SLC) pixel [13-15]. Three complex data bands HHHV, HHVV, and HVVV back scatter magnitudes are used as features for the classification. These processing steps for levee slide detection are illustrated in Figure 2. 


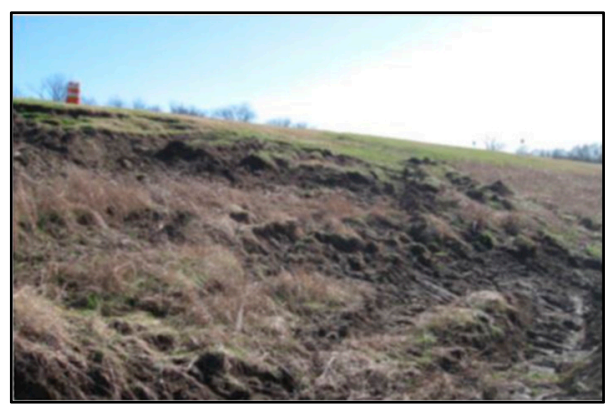

Figure 1. Slough or slump slide on a levee.

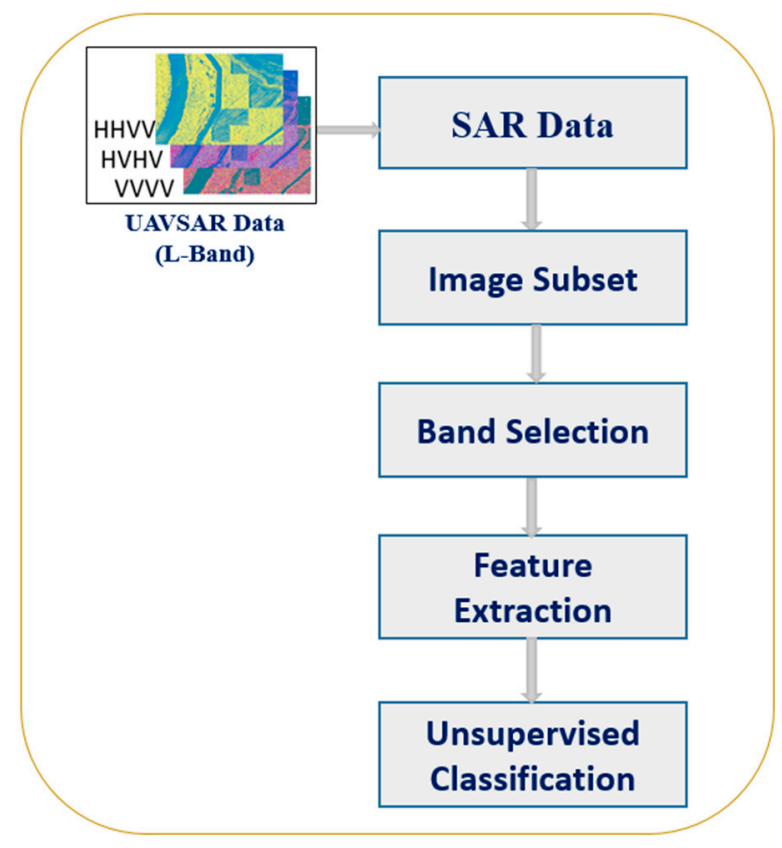

Figure 2. Processing steps for slide detection on levee.

\subsection{Data and Study Area}

The study area for this work focuses on the mainline levee system of the Mississippi River along the eastern side of the river in the state of Mississippi [16]. The fully quad-polarimetric L-band $(\lambda=23.98 \mathrm{~cm})$ SAR imagery from the NASA JPL's UAVSAR with a range bandwidth of $80 \mathrm{MHz}$ (resulting in better than 2-m range resolution) was used to detect anomalies on earthen levees. The MLC data consists of three sets of complex floating points numbers, 8 bytes per pixel. These complex products are derived from an average of 3 pixels in range and 12 pixels in azimuth, i.e., the number of range and number of azimuth looks are $3 \times 12$ of the product of each single-look complex data (SLC) pixel, which correspond to HHHV, HHVV, and HVVV. Although the raw ground sample distance is 1.6 by $0.6 \mathrm{~m}$, the multi-look $5 \times 7 \mathrm{~m}$ data is used to minimize speckle effects [5]. UAVSAR is capable of penetrating dry soil to a few centimeters depth and identifying vertical. Thus, it is valuable in detecting changes in levees that can be used as inputs to a levee monitoring system [16]. We also relied on the ground truth data collected by the US Army Corps of Engineers (USACE) which documented the location and timing of slough slide appearance and repair history. The ground truth data was also compared to optical NAIP (National Agriculture Imagery Program) imagery to visually confirm the slide events. The proposed algorithms were applied to a subset area of a levee. For the multi-polarized SAR imagery, it is useful to create a color composite image from the HH, HV, and VV channels that are being mapped to red, green, and blue, as shown in Figure 3, which includes an overview image 
overlaid on the base map. This image, collected in a single UAVSAR flight segment flying on a near north heading with the radar looking to the right, has a swath width of $20 \mathrm{~km}$ and a total length of $200 \mathrm{~km}$. Across the swath, the beam's angle of incidence varied from 20 to 65 degrees. It was collected on 25 January 2010. The study area subset is shown in Figure 3 as a red box on the radar image.

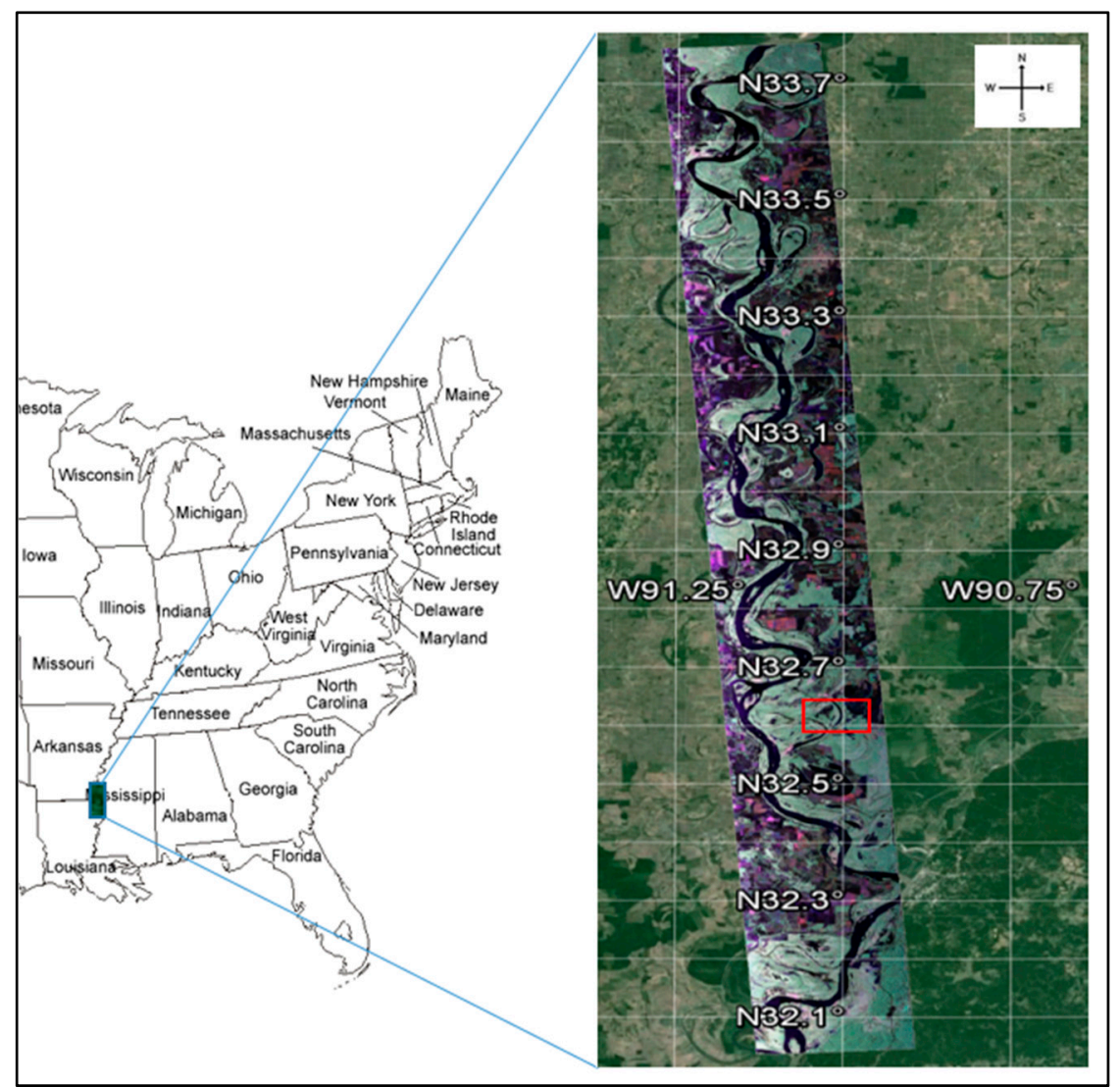

Figure 3. Study area with radar color composite 3 band (HH, VV, \& HV) image overlaid on base map.

\subsection{Ground Truth Data}

The availability of "ground truth" data is a challenge since the targets of interest are portions of levees that show signs of impending failure. Once these are detected, they are quickly repaired depending on their severity [5]. The study area is one in which the levees are managed by the US Army Corps of Engineers and are well-monitored. The Corps, in association with the local levee boards, maintains a good cumulative history of past problems and has particularly identified problematic sections of levees in the study area as shown in Table 1. In addition to the ground truth data provided by the Corps, we conducted field trips at the time of image acquisition to visually inspect the slide area and levee condition. The active slide (Slide 3) was present and unrepaired during the radar image acquisition time on 25 January 2010. Though the date of slide appearance was not identified by the Corps for Slide 3, it is visible in the NAIP (National Agriculture Imagery Program) imagery collected in 2009 and 2010 and was not repaired until after the image acquisition shown in Table 1. Hence, it was an active slide during the time of the image. 
Table 1. Updated slides ground truth from Mississippi Levee Board.

\begin{tabular}{ccccc}
\hline Slide Number & Latitude North & Longitude West & Date Slide Appeared & Date Slide Repaired \\
\hline 1 & $323637.7 \mathrm{~N}$ & $905942.3 \mathrm{~W}$ & October 2009 & November 2009 \\
2 & $323632.0 \mathrm{~N}$ & $905946.3 \mathrm{~W}$ & August 2008 & November 2009 \\
3 & $323629.1 \mathrm{~N}$ & $905948.0 \mathrm{~W}$ & - & September 2010 \\
\hline
\end{tabular}

\subsection{The $H / A / \alpha / \lambda$ Polarimetric Decomposition}

Since the multi-look samples represent spatially average values, the expected value of the $3 \times 3$ coherency matrix [T] is used to represent the averaged distributed target, as in [17]:

$$
\langle[T]\rangle=\frac{1}{N} \sum_{i=1}^{N} \underline{k}_{i} \cdot \underline{k}_{i}^{* T}=\frac{1}{N} \sum_{i=1}^{N}\left[T_{i}\right]
$$

where the symbol ${ }^{*} T$ stands for complex conjugate.

From this, the $3 \times 3$ Hermitian coherency matrix, the eigenvectors, and eigenvalues can be used to generate a diagonal form matrix that can be interpreted as the statistical independence among a set of target vectors. The coherency matrix $\langle[T]\rangle$ can then be written in this form:

$$
\langle[T]\rangle=\left[U_{3}\right][\Sigma]\left[U_{3}\right]^{-1}
$$

where $[\Sigma]$ is a $3 \times 3$ diagonal matrix with nonnegative real elements (eigenvalues) of $\langle[T]\rangle$ and $\left[U_{3}\right]=\left[\begin{array}{lll}u_{1} & u_{2} & u_{3}\end{array}\right]$ is a $3 \times 3$ unitary matrix, where $u_{1}, u_{2}$, and $u_{3}$ are the three unit orthogonal eigenvectors of $\langle[T]\rangle$, and

$$
\left[\Sigma_{3}\right]=\left[\begin{array}{ccc}
\lambda_{1} & 0 & 0 \\
0 & \lambda_{2} & 0 \\
0 & 0 & \lambda_{3}
\end{array}\right]
$$

where $\lambda_{1}>\lambda_{2}>\lambda_{3}>0$.

The polarimetric parameterization of the unit target vector $\underline{u}$ involves the combination of three simple scattering mechanisms: surface scattering, double-bounce scattering, and volume scattering, in the case of a distributed target (natural media). These can be characterized from the three components (target generators) of the unit target vector [5,7]. For the mono-static radar case, the $3 \times 3$ coherency matrix [T] has the following parameterization [7]:

$$
[T]=\underline{k} \cdot \underline{k}^{* T}=\left[\begin{array}{ccc}
2 A_{0} & C-j D & H+j G \\
C+j D & B_{0}+B & E+j F \\
H-j G & E-j F & B_{0}-B
\end{array}\right]
$$

Surface Scattering: $A_{0} \gg B_{0}+B, B_{0}-B$;

Double-bounce Scattering: $B_{0}+B \gg A_{0}, B_{0}-B$;

Volume Scattering: $B_{0}-B \gg A_{0}, B_{0}+B$.

The Cloude and Pottier [8] decomposition, based on the eigenvalue analysis of a coherency matrix, $\langle[T]\rangle$ is

$$
\langle[T]\rangle=\lambda_{1} u_{1} e_{1}^{* T}+\lambda_{2} u_{2} e_{2}^{* T}+\lambda_{3} u_{3} e_{3} * T
$$

where $\lambda_{i}$ and $u_{i}$ for $i=1,2,3$ are eigenvalues and eigenvectors.

The eigenvectors can be written as

$$
\underline{u}_{i}=\left[\cos \alpha_{i} \sin \alpha_{i} \cos \beta_{i} e^{j \delta_{i}} \sin \alpha_{i} \cos \beta_{i} e^{j \gamma_{i}}\right]^{T}
$$


Cloude and Pottier defined three parameters as a function of the eigenvalues and the eigenvectors of $\langle[T]\rangle[5,7-9]$ : entropy, $\operatorname{span}(\lambda)$, and average alpha angle.

The entropy $H$ determines the degree of statistical disorder of each target:

$$
\begin{gathered}
H=-\sum_{i=1}^{3} P_{i} \log _{3}\left(P_{i}\right), \text { and } \\
P_{i}=\frac{\lambda_{i}}{\sum_{j=1}^{3} \lambda_{j}}
\end{gathered}
$$

where $P_{i}$ is the probability of the eigenvalue $\lambda_{i}$.

The entropy, having values in the range 0 to 1 , reveals the randomness of the scattering medium, ranging from pure isotropic scattering $(H=0)$ to completely random scattering $(H=1)$. For oceans and low roughness surfaces, $H$ is near 0 , indicating the dominant mechanism is surface scattering. High values of $H$ mean multiple scattering is occurring, as in heavily vegetated areas.

Span $(\lambda)$ represents the total scattered power:

$$
\begin{gathered}
\text { span }=\left|S_{H H}\right|^{2}+\left|S_{V V}\right|^{2}+2\left|S_{H V}\right|^{2}=\sum_{i=1}^{3} \lambda_{i} \\
\text { span }=\text { Trace }\left(\left[\Sigma_{3}\right]\right)=\sum_{i=1}^{3} \lambda_{i}, \text { and } \\
\lambda=\sum_{i=1}^{3} \lambda_{i}=\lambda_{1}+\lambda_{2}+\lambda_{3}
\end{gathered}
$$

Average alpha angle $(\alpha)$ identifies the dominant scattering mechanism for different scattering processes:

$$
\alpha=\sum_{i=1}^{3} P_{i} \alpha_{i}=P_{1} \alpha_{1}+P_{2} \alpha_{2}+P_{3} \alpha_{3}
$$

$\alpha$ reveals the averaged scattering mechanisms from surface scattering $(\alpha=0)$ to double bounce $(\alpha=90)$.

\subsection{Unsupervised $\mathrm{H} / \alpha$ Classification}

Unsupervised classification schemes were implemented using $\mathrm{H}$ and $\alpha$. All random scattering process can be represented in this 2-dimensional feature space. The underlying principle is that entropy is an indicator of the reversibility of the scattering, while the angle $(\alpha)$ is related to the average scattering mechanism present [17]. The classification plane is divided into nine different zones that represent the different scattering processes, as shown in Figure 4. The classification method is implemented by comparing the observed values of $\mathrm{H}$ and $\alpha$ to these fixed zone thresholds to identify the scattering mechanism. The value of $\alpha$ segments the plane into regions characteristic of surface, volume, or multiple scattering. The H or entropy value separates regions of low, medium, and high amounts of randomness along the $x$ axis $[9,17,18]$. The corresponding net combined physical scattering characteristics of each of the zones thus become as follows [9]: Z9: Low Entropy Surface Scattering; Z8: Low Entropy Dipole Scattering; Z7: Low Entropy Multiple Scattering; Z6: Medium Entropy Surface Scattering; Z5: Medium Entropy Vegetation Scattering; Z4: Medium Entropy Multiple Scattering; Z3: (Not a Feasible Region); Z2: High Entropy Vegetation Scattering; and Z1: High Entropy Multiple Scattering. 


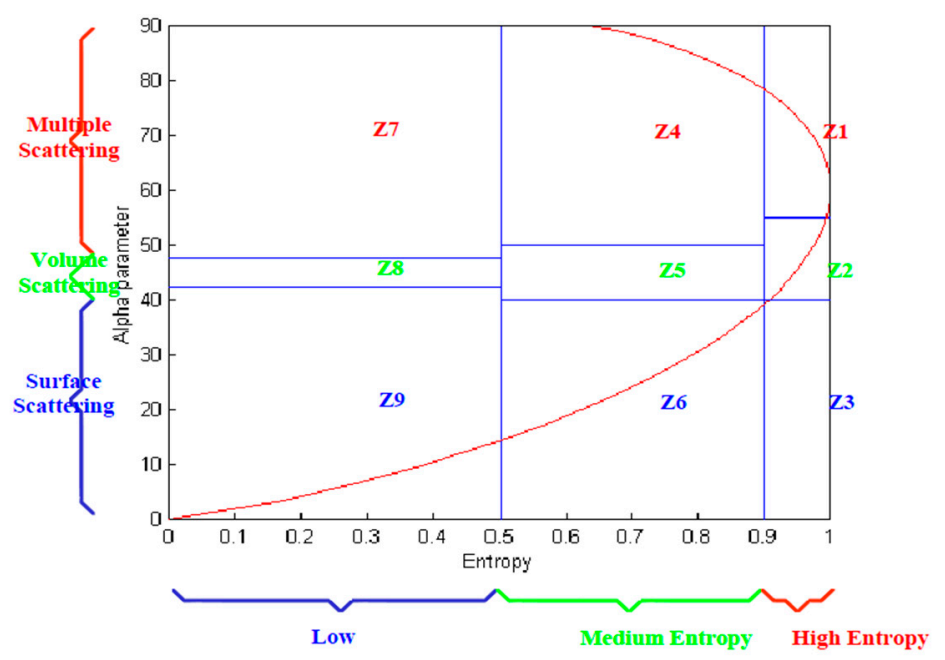

Figure 4. Segmentation of the $\mathrm{H} / \alpha$ space [19].

A refinement of this simple classification was applied next using the method of Lee et al. [12]. The initial classification map from the fixed-zone $\mathrm{H} / \alpha$ plane defines training sets for a maximum likelihood classification based on the assumption that the data follow a Wishart distribution and the use of the coherency matrix elements as the features. The class of each resulting cluster is determined from whichever zone of the $\mathrm{H} / \alpha$ plane the new class center falls in. The classified results are then used as training sets for the next iteration, repeating until a threshold for the percentage of pixels switching classes or an iteration count is met.

\section{5. $H / A / \alpha$ Unsupervised Classification}

A further refinement of the Wishart-based $\mathrm{H} / \alpha$ segmentation makes use of an anisotropy parameter during the procedure. This parameter indicates the relative significance of secondary scattering processes. It allows the discrimination of scattering mechanisms having similar entropy values but different eigenvalue distributions. In these cases, greater anisotropy indicates the presence of two dominant scattering processes having equal probability and a less important third mechanism, while low anisotropy reveals a dominant primary scattering process and two secondary mechanisms that are not negligible and have equal significance [17-19]. Polarimetric data is first segmented using the maximum likelihood Wishart method. After this procedure is complete, the 8 resulting segments are refined into 16 based on the anisotropy value of each pixel, using a fixed threshold of 0.5 . The 16 resulting clusters are then used as training for a second Wishart maximum likelihood classification. The use of anisotropy in the segmentation process allows the splitting of large segments into smaller ones that discriminate small differences in a refined manner, grouping pixels together with similar statistics $[14,17,18]$.

\section{6. $H / \alpha / \lambda$ Unsupervised Classification}

Cloude and Pottier [9] demonstrated an unsupervised classification based on the $\mathrm{H} / \alpha$ parameters. These parameters alone were not sufficient for good interclass resolution, indicating that additional information is needed. Even though the $\mathrm{H}$ and $\alpha$ values are derived from fully polarimetric data, they do not completely represent all the polarimetric information. Other parameters, including the span or specific correlation coefficients were expected to significantly improve the classification [20]. Hellmann et al. [11,15] tested an unsupervised classification based on the $H / \alpha / \lambda_{1}$ parameters. However, $\lambda_{1}$ alone was not able to represent the complete scattering mechanism about the target. The $\mathrm{H} / \alpha / \lambda$ classification including classification for individual $\lambda$ values such as $H / \alpha / \lambda_{1}$ is performed for good interclass resolution [12,18]. We implemented classification using $H / \alpha / \lambda, H / \alpha / \lambda_{1}, H / \alpha / \lambda_{2}$, and 
$H / \alpha / \lambda_{3}$. In the $H / \alpha / \lambda$ approach, the backscatter intensity information contained in the eigenvalues $\lambda_{1}, \lambda_{2}$, and $\lambda_{3}$ is used to improve the interclass resolution due to the differing reflectivities of different scatterers.

\section{Results and Discussion}

The motivation of this work is to detect slough slides on a levee using remotely sensed imagery. PolSAR data was used for classification of scattering mechanisms of a target, such as surface, double-bounce, or volume scattering [18]. The yellow line overlaid on the optical image in Figure 5 shows the analysis area of the levee from the river side toe to the center line (crown) of the levee. At present, we are focusing on the river side because the chance of the occurrence of slough sides is significantly greater there compared to the land side of the levee. On this image, the locations of the three slides are also indicated.

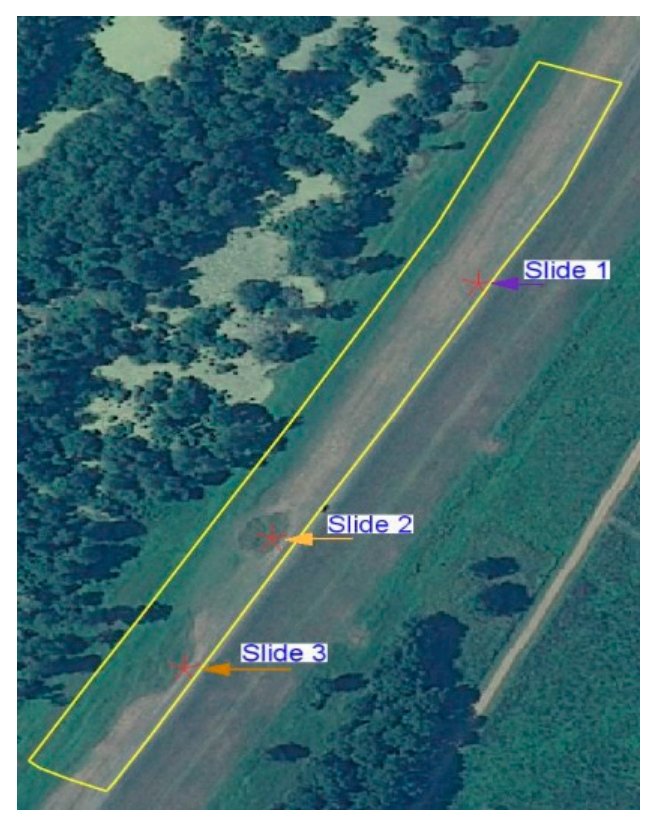

Figure 5. Optical image overlaid with the test area in yellow.

Figure 6a shows the radar backscatter image in the Pauli color composite form (red $=\mathrm{HH}+\mathrm{VV}$, green $=\mathrm{HH}-\mathrm{VV}$, and blue $=\mathrm{HV}$ ). In Figure $6 \mathrm{~b}-\mathrm{f}$, we see respectively the output class map from each of the following classifiers: $\mathrm{H} / \alpha$, Wishart- $\mathrm{H} / \alpha, \mathrm{H} / \mathrm{A}, \mathrm{A} / \alpha$, and Wishart-H/A/ $\alpha$ classification. The $\mathrm{H} / \alpha, \mathrm{H} / \mathrm{A}, \mathrm{A} / \alpha$, and Wishart-H/ $\alpha$ classifiers generate nine classes, based on the use of the two-dimensional $\mathrm{H} / \alpha$ classification plane. Wishart-H/A/ $\alpha$ classification produces 16 classes based on the $\mathrm{H} / \mathrm{A} / \alpha$ segmentation plane. For the Wishart-H/ $\alpha$ and Wishart-H/A $/ \alpha$ classification, the polarimetric decomposition parameters (entropy, alpha, and anisotropy) were used as training sets in these iterative classification algorithms. The iteration halted after 10 cycles, when the fraction of class switching was under $10 \%$. Figure 7 repeats the two clearly superior class maps among these-the two Wishart classifier maps-showing how they align with the locations of slides, as shown in the optical image in Figure 7c. 


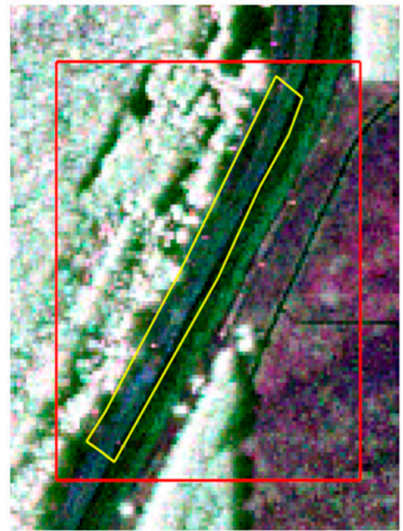

(a)
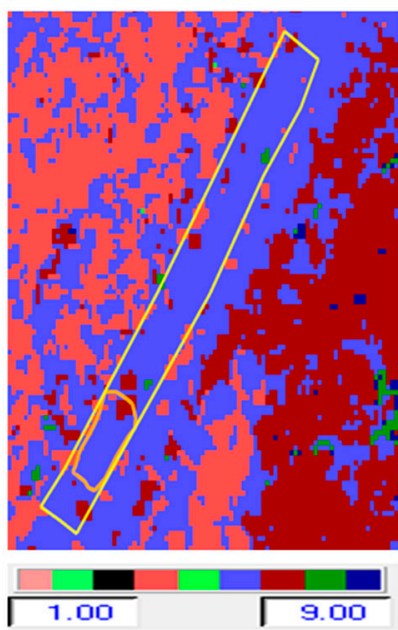

(d)
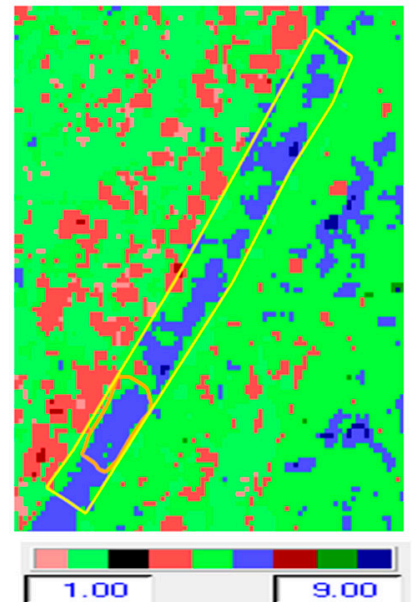

(b)

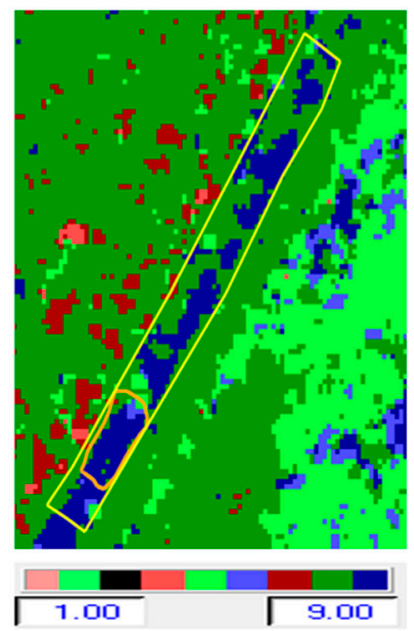

(e)
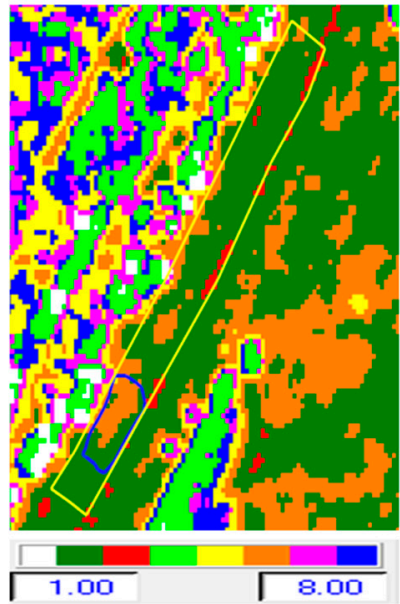

(c)

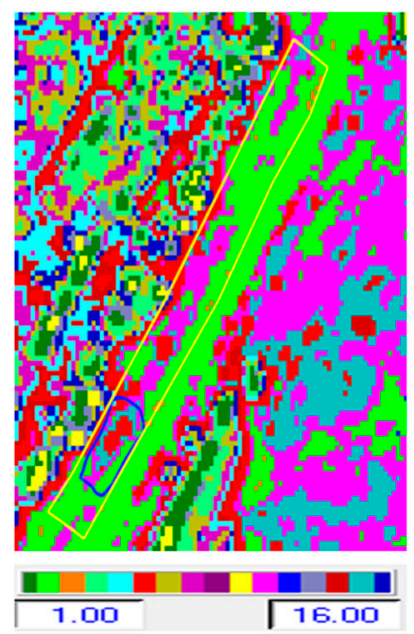

(f)

Figure 6. (a-c) Pauli RGB Image, $\mathrm{H} / \alpha$ classification, and Wishart-H/ $\alpha$ classification; (d-f) H/A classification, A/ $\alpha$ classification, and Wishart-H/A/ $\alpha$ classification.

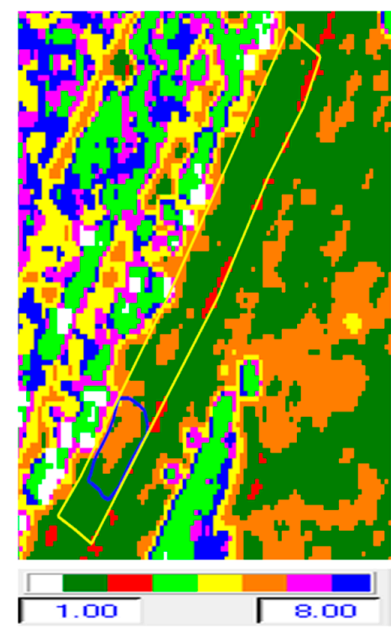

(a)

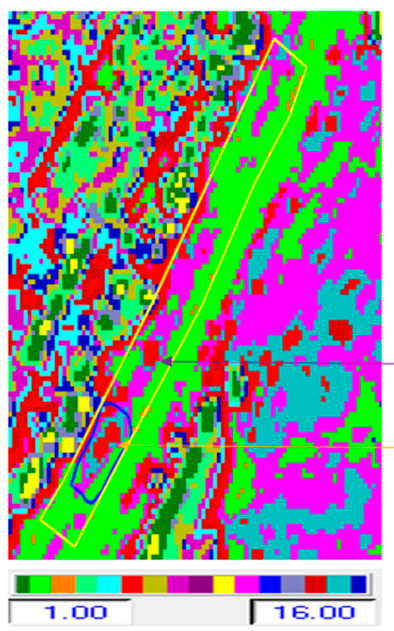

(b)

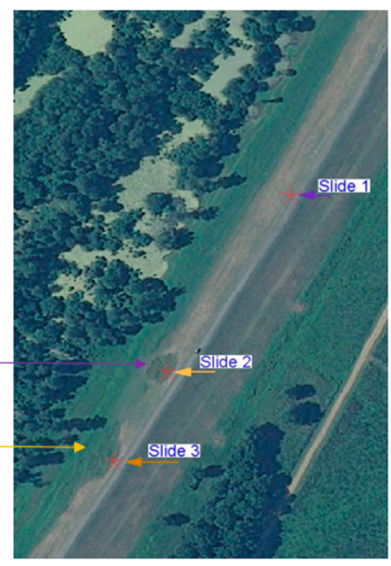

(c)

Figure 7. (a-c) Wishart-H/ $\alpha$ classification, Wishart-H/A/ $\alpha$ classification, and optical image. 
Figure 8a-f shows: (a) The Pauli RGB image; (b) $\mathrm{H} / \alpha$ classification (repeated for comparison); (c) $\mathrm{H} / \alpha / \lambda$ classification; (d) $\mathrm{H} / \alpha / \lambda_{1}$ classification; (e) $\mathrm{H} / \alpha / \lambda_{2}$ classification; and (f) $\mathrm{H} / \alpha / \lambda_{3}$ classification. The latter three classifications are inter-classified within the $9 \mathrm{H} / \alpha$ to represent the interclass resolution due to the different reflectivities of different scatterers [11,15]. All the class values of segmented zones for the $H / \alpha, H / \alpha / \lambda_{1}, H / \alpha / \lambda_{2}$, and $H / \alpha / \lambda_{3}$ classifications using the $\mathrm{H} / \alpha$ segmentation plane for random media scattering are listed in Table 2 . Using these values, the classification color map representing each class of $\mathrm{H} / \alpha / \lambda$ classification was extended from 9 colors to 27 colors. In the classification with individual eigenvalue analysis, the $H / \alpha / \lambda_{1}$ classification shows where surface scattering dominates; the $\mathrm{H} / \alpha / \lambda_{2}$ classification highlights areas dominated by double-bounce scattering. In the $\mathrm{H} / \alpha / \lambda_{3}$ classification, the volume scattering is emphasized. For the slough slide areas, it can be seen that the surface scattering is partially dominant, the double-bounce scattering is strongly dominant, and the volume scattering is almost zero, since here our target (levee) is naturally distributed.

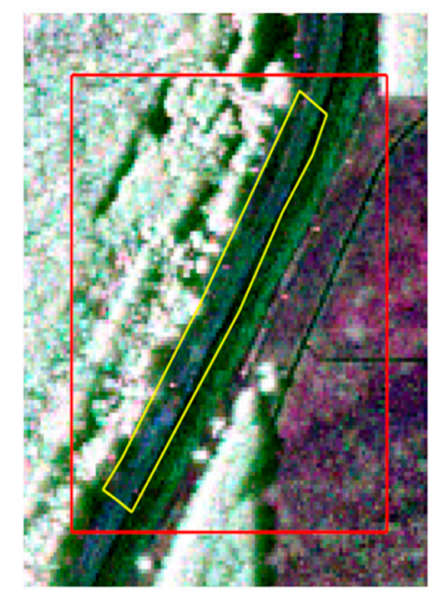

(a)

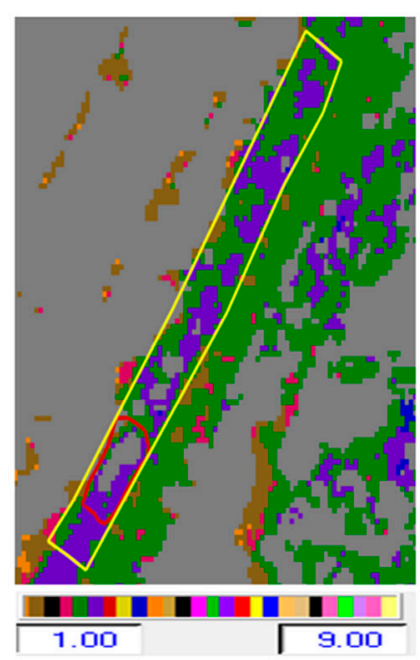

(d)

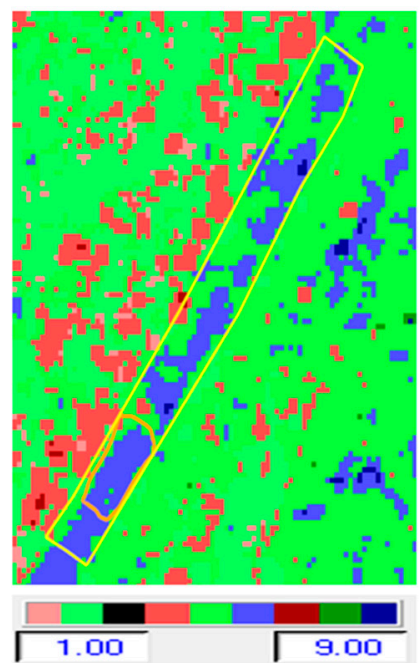

(b)

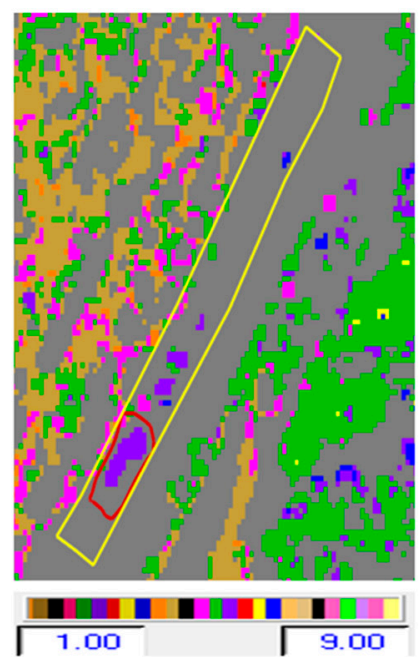

(e)

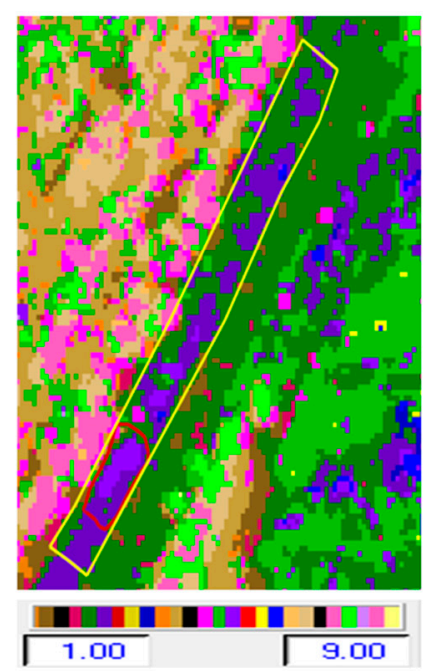

(c)
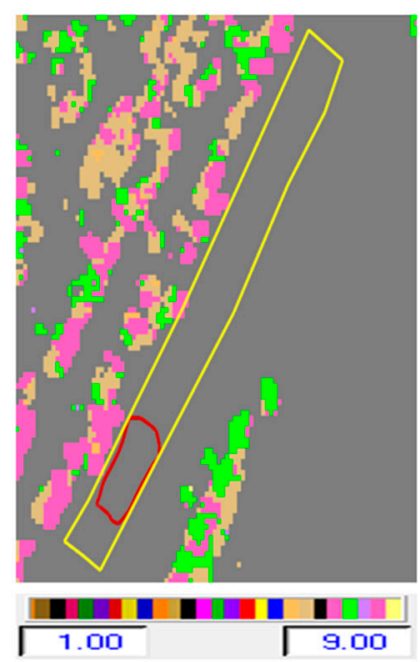

(f)

Figure 8. (a-c) Pauli RGB Image, $H / \alpha$ classification, and $H / \alpha / \lambda$ classification; $(\mathbf{d}-\mathbf{f}) H / \alpha / \lambda_{1}$ classification, $H / \alpha / \lambda_{2}$ classification, and $H / \alpha / \lambda_{3}$ classification. 
Table 2. Class values of segmented zones for the $H / \alpha, H / \alpha / \lambda_{1}, H / \alpha / \lambda_{2}$, and $H / \alpha / \lambda_{3}$ classifications using the $\mathrm{H} / \alpha$ segmentation plane for random media scattering.

\begin{tabular}{ccccc}
\hline \multirow{2}{*}{ Zone/Class Value } & \multicolumn{4}{c}{ Classification } \\
\cline { 2 - 5 } & $\mathbf{H} / \boldsymbol{\alpha}$ & $\mathbf{H} / \boldsymbol{\alpha} / \boldsymbol{\lambda}_{\mathbf{1}}$ & $\mathbf{H} / \boldsymbol{\alpha} / \boldsymbol{\lambda}_{\mathbf{2}}$ & $\mathbf{H} / \boldsymbol{\alpha} / \boldsymbol{\lambda}_{\mathbf{3}}$ \\
\hline Z1 & 1 & 1 & 3.37 & 6.54 \\
Z2 & 2 & 1.31 & 4.08 & 6.85 \\
Z3 & 0 & 0 & 0 & 0 \\
Z4 & 4 & 1.92 & 4.69 & 8.38 \\
Z5 & 5 & 2.23 & 5.00 & 7.77 \\
Z6 & 6 & 2.54 & 5.31 & 8.08 \\
Z7 & 7 & 2.85 & 5.62 & 8.38 \\
Z8 & 8 & 3.15 & 5.92 & 8.69 \\
Z9 & 9 & 3.46 & 6.23 & 0 \\
\hline
\end{tabular}

The slough slide area is marked with a polygon (found in the southern end of the test area) and the test area (on the river side of the levee) is outlined in yellow in the figures. The locations of the three slides are indicated on the optical image with red stars. For this subset, although some of the slide areas (slides 1 and 2) had been repaired by the time of image acquisition, they still show up as anomalies detected by the classification techniques to some extent as shown in Figures $7 \mathrm{a}-\mathrm{c}$ and $9 \mathrm{a}-\mathrm{c}$. Because these slide areas were repaired only two months prior to the time of image acquisition, they still appear anomalous because of the surface roughness and differences in the grass cover. Generally, the healthy levee area has a uniform pattern, but the slide areas have a different pattern in the radar backscattering data [21]. Sometimes other artifacts show similar patterns as the slide area. An example of this is highlighted by the yellow arrow in Figure 9b,c, which is an area influenced by a tall tree nearby casting a radar shadow on the levee. False positives may also occur in the classification process due to rough non-slide surfaces or other anomalies. Specifically, the presence of some anomalous areas in the vicinity of the slide areas may be due to the similarity of soil properties or the vegetation type and condition there, as was verified using in situ measurements of soil properties in [22].

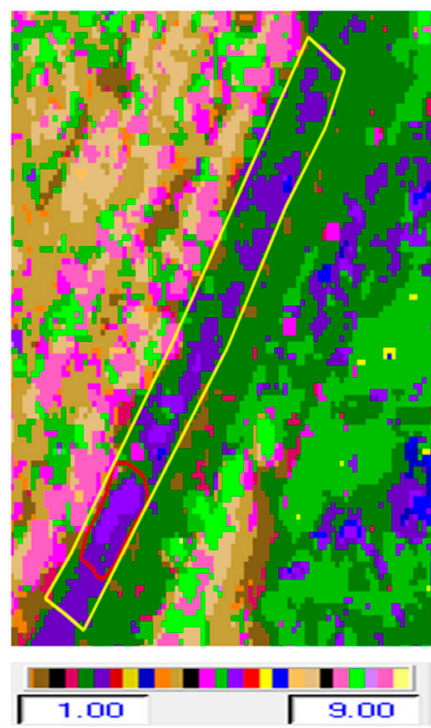

(a)

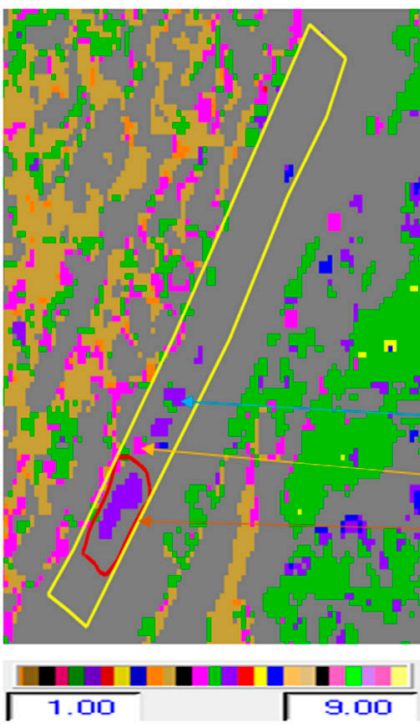

(b)

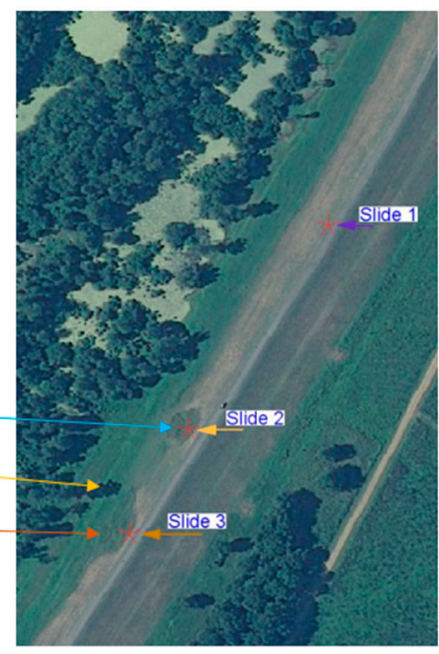

(c)

Figure 9. (a-c) $H / \alpha / \lambda$ classification, $H / \alpha / \lambda_{2}$ classification, and optical image.

A qualitative assessment of the classification results reveals that the Wishart- $\mathrm{H} / \alpha$, Wishart-H/A/ $\alpha$, and $H / \alpha / \lambda$ methods provide superior classification for this application compared 
with the other unsupervised schemes tested. The discrimination of the slide and related anomalies from "healthy" levee areas was effective and was improved by incorporating more parameters. The detected slide and anomalies in the classification results are compared with the optical NAIP image in Figures $7 \mathrm{a}-\mathrm{c}$ and $9 \mathrm{a}-\mathrm{c}$.

The $\mathrm{H} / \alpha$ classification uses nine classes, as shown in Figure 6b: out of those, only 4 classes (green, light blue, red, and dark blue) are found in the test area. Other classes occur outside the levee area. This $\mathrm{H} / \alpha$ classification does not effectively discriminate the slide area from the non-slide areas. Similarly, the H/A classification and A/ $\alpha$ classification result in nine classes as shown in Figure $6 \mathrm{~d}, \mathrm{e}$, only four of which are found in the test area. The segmented and occurrence planes for the $H / \alpha, H / A$, and $\mathrm{A} / \alpha$ classifications are shown in Figure 10a-f. Once again, the likelihood of identifying slides from this data is not good. This motivated the inclusion of Wishart-based classification, in which the initial classification map defines training sets for classification based on the Wishart distribution iteratively. Significant improvement in each iteration was observed, and the analysis of the final class centers in the two-dimensional $\mathrm{H} / \alpha$ classification plane is used for the identification of slides. The Wishart-H/ $\alpha$ classification uses eight classes, as shown in Figure 6c, of which for the most part only two classes (green and orange) are found in the test area on the levee. These two classes exhibit good discrimination between slide and nonslide (healthy) areas of the levee.

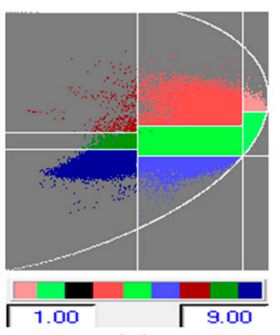

(a)

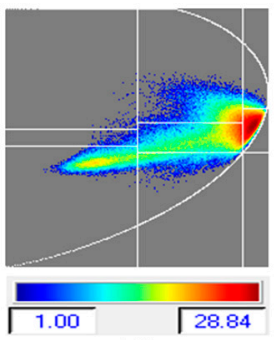

(d)

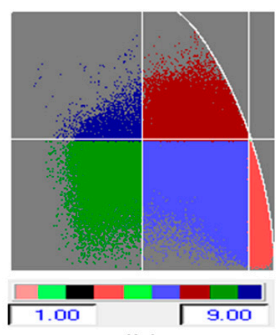

(b)

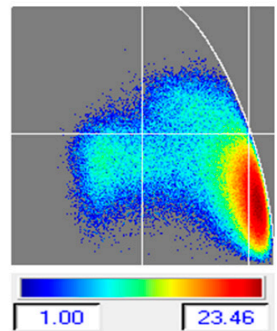

(e)

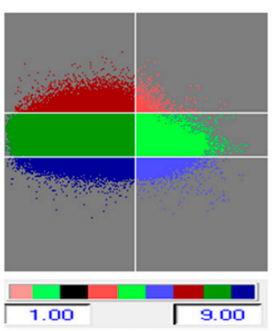

(c)

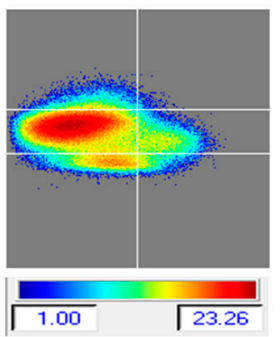

(f)

Figure 10. (a-c) segmented planes for $\mathrm{H} / \alpha, \mathrm{H} / \mathrm{A}$, and $\mathrm{A} / \alpha$ classification; $(\mathbf{d}-\mathbf{f})$ occurrence planes for $\mathrm{H} / \alpha, \mathrm{H} / \mathrm{A}$, and $\mathrm{A} / \alpha$ classification.

The Wishart-H/A/ $\alpha$ classification is based on 16 classes, as shown in Figure 6f. Out of those, four (colored parrot green, pink, red, and dark ash) occur in the test area. These four classes clearly discriminate the slide and nonslide areas of the levee, as well as distinguishing the area near the slide from other healthy areas on the levee. The segmented and occurrence planes for $H / \alpha / \lambda_{1}, H / \alpha / \lambda_{2}$, and $\mathrm{H} / \alpha / \lambda_{3}$ classifications are shown in Figure 11a-f. For the slough slide areas, once again it can be seen that the surface scattering is partially dominant, the double-bounce scattering is strongly dominant, and the volume scattering is almost zero. The $\mathrm{H} / \alpha / \lambda$ classification and $\mathrm{H} / \alpha / \lambda_{2}$ classification clearly identified the slide/anomalous areas, as highlighted in Figure $8 \mathrm{c}, \mathrm{e}$. The polarimetric SAR data processing and educational tool (PolSARpro v4.2.0 software) from the European Space Agency was used for this work [23]. 


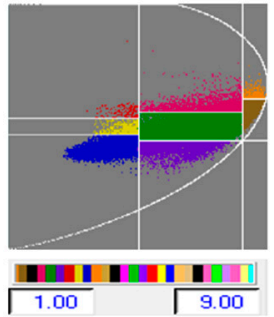

(a)

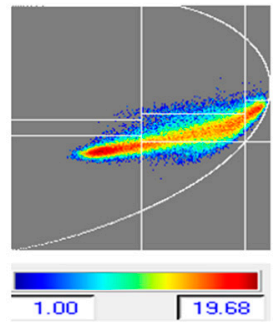

(d)

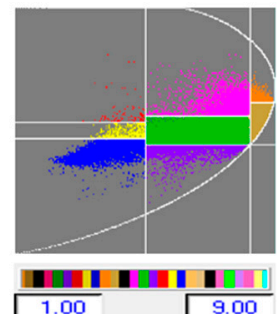

(b)

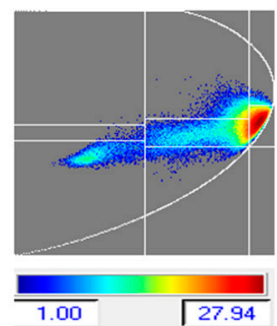

(e)

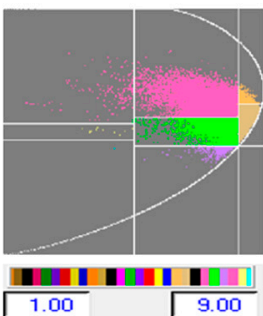

(c)

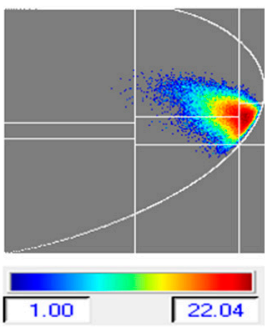

(f)

Figure 11. (a-c) segmented planes for $H / \alpha / \lambda_{1}, H / \alpha / \lambda_{2}$, and $H / \alpha / \lambda_{3}$ classification; (d-f) occurrence planes for $H / \alpha / \lambda_{1}, H / \alpha / \lambda_{2}$, and $H / \alpha / \lambda_{3}$ classification.

Although formal quantitative accuracy assessment cannot be performed on unsupervised results without first labeling the output clusters-which is typically done using manual interpretation-we can somewhat quantify the accuracy of this result by noting that a single output cluster dominated the area of the active slide (i.e., Slide 3) and can use it to estimate detection accuracy. In this case, $96 \%$ of the slide pixels were detected as such, and as can be seen in Figure 9b, there were very few false positives based on this labeling. Furthermore, almost all of the false positives fall in the area of the recently repaired Slide 2.

\section{Conclusions}

This work presents the results of using SAR data to detect anomalies on an earthen levee. Unsupervised $\mathrm{H} / \alpha, \mathrm{H} / \mathrm{A}, \mathrm{A} / \alpha$, Wishart $\mathrm{H} / \alpha$, Wishart $\mathrm{H} / \mathrm{A} / \alpha$, and $\mathrm{H} / \alpha / \lambda$ (also individually including $\lambda_{1}, \lambda_{2}$, and $\lambda_{3}$ ) classification algorithms were applied to polarimetric SAR data. The effectiveness of the algorithms is demonstrated using fully quad-polarimetric L-band SAR imagery from the NASA JPL's UAVSAR. The study area is a section of the lower Mississippi River valley in the Southern USA.

Results show that slough slides on levees exhibit distinctive scattering mechanisms compared with the healthy (i.e., nonslide) areas, and that these differences are revealed by unsupervised classification methods utilizing the polarimetric decomposition parameters $\mathrm{H}, \mathrm{A}, \alpha$, and $\lambda$. The resulting color-coded class maps can be used to detect anomalous areas on the levee for closer inspection. Wishart-based unsupervised classification schemes clearly show better results for this application. Furthermore, $\mathrm{H} / \alpha / \lambda_{2}$ classification shows noticeably better results to identify slough slide areas. The results indicate that, on the levee, slide areas scatter predominantly as double bounce; meanwhile, in other healthy parts of the levee, surface scattering dominates.

In addition to the active slide area, other anomalous areas are also detected. One interesting point that we noticed is that some of the slide areas that had been repaired just two months prior to the time of image acquisition still appear anomalous because of the texture roughness and differences in grass thickness, and are detected by the classification technique. To validate the attribution of scattering mechanisms such as these to the different surface classes, model-based polSAR decompositions can be used. Early results of that approach were reported in [24]. 
Although the test study area is small, including only a single active slide area, the methods presented in this paper show promising results. Planned future work includes the use of larger test areas consisting of more active slides, seasonal images acquired by the polSAR, and different geometrical orientations of the levee.

Acknowledgments: This material is based upon work supported by the National Science Foundation under Award No. OISE-1243539 and by the NASA Applied Sciences Division under grant number NNX09AV25G. The authors would like to thank the US Army Corps of Engineers, Engineer Research and Development Center and Vicksburg Levee District for providing ground truth data and expertise, the NASA Jet Propulsion Laboratory for providing the UAVSAR image, and the GRI levee team.

Author Contributions: Ramakalavathi Marapareddy implemented the classification methods on image processing tools. James V. Aanstoos supervised the work, provided imagery and data and was the principal investigator for the project. Nicolas H. Younan supervised and provided guidance. Ramakalavathi Marapareddy, James V. Aanstoos, and Nicolas H. Younan analyzed the results and wrote the paper.

Conflicts of Interest: The authors declare no conflict of interest.

\section{References}

1. Aanstoos, J.V.; Hasan, K.; O’Hara, C.G.; Prasad, S.; Dabbiru, L.; Mahrooghy, M.; Nobrega, R.; Lee, M.L.; Shrestha, B. Use of Remote Sensing to Screen Earthen Levees. In Proceedings of the 39th Applied Imagery Pattern Recognition Workshop (AIPR), Washington, DC, USA, 13-15 October 2010; pp. 1-6.

2. Dunbar, J. USACE's Lower Mississippi Valley Engineering Geology and Geomorphology Mapping Program for Levees; USACE: Vicksburg, MS, USA, 2009.

3. Hossain, A.K.M.A.; Easson, G.; Hasan, K. Detection of Levee Slides Using Commercially Available Remotely Sensed Data. Environ. Eng. Geosci. 2006, 12, 235-246. [CrossRef]

4. Seed, R.B.; Bea, R.G.; Independent Levee Investigation Team. Investigation of the Performance of the New Orleans Flood Protection Systems in Hurricane Katrina on 29 August 2005. Final Report, Volume 1, 31 July 2006. Available online: http:/ /www.ce.berkeley.edu/ \{\}new_orleans / (accessed on 14 June 2016).

5. Aanstoos, J.V.; Hasan, K.; O’Hara, C.; Dabbiru, L.; Mahrooghy, M.; Nobrega, R.A.A.; Lee, M.M. Detection of Slump Slides on Earthen Levees Using Polarimetric SAR Imagery. In Proceedings of the Conference: 2012 IEEE Applied Imagery Pattern Recognition Workshop, Washington, DC, USA, 9-11 October 2012.

6. Ouchi, K. Recent Trend and Advance of Synthetic Aperture Radar with Selected Topics. Remote Sens. 2013, 5, 716-807. [CrossRef]

7. Ainsworth, T.L.; Cloude, S.R.; Lee, J.S. Eigenvector analysis of polarimetric SAR data. In Proceedings of the IEEE International Geoscience and Remote Sensing Symposium, Toronto, ON, Canada, 24-28 June 2002; Volume 1, pp. 626-628.

8. Cloude, S.R.; Pottier, E. A review of target decomposition theorems in radar polarimetry. IEEE Trans. Geosci. Remote Sens. 1996, 34, 498-518. [CrossRef]

9. Cloude, S.R.; Pottier, E. An entropy based classification scheme for land applications of polarimetric SAR. IEEE Trans. Geosci. Remote Sens. 1997, 35, 68-78. [CrossRef]

10. Cloude, S.R.; Pottier, E.; Boerner, W.M. Unsupervised image classification using the entropy/alpha/ anisotropy method in radar polarimetry. In Proceedings of the NASA-JPL, AIRSAR-02 Workshop, Pasadena, CA, USA, 4-6 March 2002; pp. 4-6.

11. Hellmann, M.; Jager, G.; Kratzschmar, E.; Habermeyer, M. Classification of full polarimetric SAR-data using artificial neural networks and fuzzy algorithms. In Proceedings of the IEEE International Geoscience and Remote Sensing Symposium, Hamburg, Germany, 28 June-2 July 1999; Volume 4, pp. 1995-1997.

12. Lee, J.S.; Grunes, M.R.; Anisoworth, T.L.; Du, L.J.; Schuler, D.L.; Coulde, S.R. Unsupervised classification using polarimetric decomposition and the complex Whishart classifier. IEEE Trans. Geosci. Remote Sens. 1999, $35,2249-2258$.

13. Lee, J.S.; Grunes, M.R. Classification of multi-look polarimetric SAR data based on complex Wishart distribution. In Proceedings of the IEEE National Telesystems Conference, Washington, DC, USA, 19-20 May 1992; pp. 7-21. 
14. Pottier, E.; Lee, J.S. Application of the H/A/alpha Polarimetric Decomposition Theorem for Unsupervised Classification of Fully Polarimetric SAR Data Based on the Wishart Distribution. In Proceedings of the SAR Workshop: CEOS Committee on Earth Observation Satellites, Working Group on Calibration and Validation, Toulouse, France, 26-29 October 1999.

15. Hellmann, M.; Jager, G.; Pottier, E. Fuzzy clustering and interpretation of fully polarimetric SAR data. In Proceedings of the IEEE International Geoscience and Remote Sensing Symposium, Sydney, NSW, Australia, 9-13 July 2001; Volume 6, pp. 2790-2792.

16. Aanstoos, J.V.; Dabbiru, L.; Gokaraju, B.; Hasan, K.; Lee, M.A.; Mahrooghy, M.; Nobrega, R.A.A.; O'Hara, C.G.; Prasad, S.; Shanker, A. Levee Assessment via Remote Sensing; SERRI Report 80023-02; Southeast Region Research Initiative: Oak Ridge, TN, USA, 2012.

17. Pottier, E.; Lee, J.S.; Laurent, F.F. Polarimetric SAR Data Processing and Educational Tool Tutorial Lecture Notes on Advanced Concepts. I.E.T.R-UMR CNRS 6164-University of Rennes1 and Naval Research Laboratory, at the University of Rennes 1, DLR-HR. Available online: https:/ / earth.esa.int/web/polsarpro/ polarimetry-tutorial (accessed on 1 November 2014).

18. Freeman, A.; Durden, S.T. A Three-Component Scattering Model for Polarimetric SAR Data. IEEE Trans. Geosci. Remote Sens. 1998, 36, 963-973. [CrossRef]

19. Boerner, W.M. Polarimetric SAR Data Processing and Educational Tool Tutorial Lecture Notes on Basic Concepts in Radar Polarimetry. UIC-ECE Communications, Sensing \& Navigation Laboratory, at the University of Rennes 1, DLR-HR. Available online: https://earth.esa.int/web/polsarpro/polarimetrytutorial (accessed on 1 November 2014).

20. Chan, Y.K.; Koo, V.C. An Introduction to Synthetic Aperture Radar (SAR). Prog. Electromagn. Res. B 2008, 2, 27-60. [CrossRef]

21. Singhroy, V.H.; Loehr, J.E.; Correa, A.C. Landslide risk assessment with high spatial resolution remote sensing satellite data. In Proceedings of the IEEE International Geoscience and Remote Sensing Symposium, IGARSS, Honolulu, HI, USA, 24-28 July 2000; Volume 6, pp. 2501-2503.

22. Sehat, S.; Vahedifard, F.; Aanstoos, J.V.; Dabbiru, L.; Hasan, K. Using in situ Soil Measurements for Analysis of a Polarimetric SAR-based Classification of Levee Slump Slides in the Lower Mississippi River. Eng. Geol. 2014, 181, 157-168. [CrossRef]

23. PolSARpro (Polarimetric SAR Data Processing and Educational Tool v4.0) Software. Available online: https: / / earth.esa.int/web/polsarpro/download/release-notes/previous-versions/-/article/version-4-15-to-version-4-2 (accessed on 1 November 2014).

24. Ramakalavathi, R.; Aanstoos, J.V.; Younan, N.H. Unsupervised classification of SAR imagery using polarimetric decomposition to preserve scattering characteristics. In Proceedings of the IEEE Applied Imagery Pattern Recognition Workshop (AIPR), Washington, DC, USA, 13-15 October 2015; pp. 1-5. [CrossRef]

(C) 2016 by the authors; licensee MDPI, Basel, Switzerland. This article is an open access article distributed under the terms and conditions of the Creative Commons Attribution (CC-BY) license (http://creativecommons.org/licenses/by/4.0/). 